\title{
Die Afrikanische Charta der Menschenrechte und Rechte der Völker zwischen afrikanischen Wertvorstellungen und der Bindung an das internationale Recht: Das Beispiel des Art. 18 Abs. 1- 3 AfrC
}

\author{
Von Michaela Wittinger
}

\section{Einleitung und Fragestellung}

Die Afrikanische Charta der Menschenrechte und Rechte der Völker ${ }^{1}$ ist wie die Europäische Menschenrechtskonvention ${ }^{2}$ und die Amerikanische Menschenrechtskonvention ${ }^{3}$ ein regionales Menschenrechtsinstrument. Anders als die beiden Konventionen, die vorrangig die "klassischen" bürgerlichen und politischen Grundrechte des Individuums garantieren, verbrieft die Charta auch wirtschaftliche und soziale Rechte. Ferner enthält sie nicht nur Rechte des Einzelnen sowie Rechte der Völker, sondern berücksichtigt auch gesellschaftliche Gruppen, z.B. Familien und Frauen. ${ }^{4}$ Institutionell unterscheidet sich die Afrikanische Charta - noch - deutlich von ihren beiden "Schwestern", da sie bisher nur über ein nicht judikatives Organ, die Afrikanische Kommission der Menschenrechte und Rechte der Völker ${ }^{5}$, verfügt. Ein Afrikanischer Gerichtshof soll aber geschaffen werden, das entsprechende Protokoll liegt vor. ${ }^{6}$

Im folgenden: AfrC, v. 27.6.1981, in Kraft seit 21.10.1986, OAU/Doc.CAB/LEG/67/3/Rev. 5., frz. Fassung in Revue Universelle des Droits de l'Homme 1 (1989), S. 484 ff.; engl. z.B. in International Legal Materials 21 (1982), S. 59 ff.; deutsche Übers. z.B. in Europäische Grundrechtszeitschrift 13 (1986), S. 677 ff. Bindend ist nur die engl. und frz. Version.

Im folgenden: EMRK, v. 4.11.1950, geänd. durch die ZPe 3, 5, 8, 9, UNTS Bd. 213, S. 221 ff.; BGBl. 1952 II, S. 686 ff., 931; 1968 II S. 116, 1120, 1989 II S. 547 und durch das Reformprotokoll Nr. 11, v. 11.5.1994, abgedruckt in EuGRZ 21 (1994), S. 323 ff., in Kraft seit dem 1.11.1998.

Im folgenden: AMRK, in Kraft seit 18.7.1978; OAS Official Records OEA/Ser.K/XVI/1.1., Doc. 65, Rev. 1, Corr. 1 of January 7, 1970, z.B. in: Buergenthal / Shelton, Protecting Human Rights in the Americas, Cases and Materials (Kehl et al., 4. Aufl. 1995), S. 617 ff.; deutsche Übers. in Europäische Grundrechtszeitschrift 7 (1980), S. 435 ff.

Art. 18 Abs. 1, 2 und 3 AfrC.

5 Im Folgenden: AfrKMRV.

6

Protocol to the African Charter on Human and Peoples' Rights on the Establishment of an African Court on Human and Peoples' Rights v. 9.6.1998, in Human Rights Law Journal 20 (1999), S. 727 ff., noch nicht in Kraft, hierzu Marie, Human Rights Law Journal 20 (1999), S. 
Im Unterschied zu den beiden anderen regionalen Konventionen betont die Charta ihre afrikanische Identität und unterstreicht mehrfach "die traditionellen afrikanischen Werte". 7 Sehr deutlich wird dies in Art. 18 Abs. 2 AfrC, der den Staat verpflichtet, die Familie "als Bewahrerin der Sittlichkeit und der in der Gemeinschaft anerkannten traditionellen Werte zu unterstützen" ("d'assister la famille dans sa mission de gardienne de la morale et des valeurs traditionnelles reconnues par la Communauté / to assist the family, which is the custodian of morals and traditional values recognized by the community"). Zugleich sieht sich die Afrikanische Charta dem internationalen Recht verpflichtet. ${ }^{8}$ Besondere Bedeutung kommt dem Verweis auf internationales Recht in Art. 18 Abs. 3 AfrC zu, wonach "die Rechte der Frau ..., wie sie in internationalen Konventionen und Deklarationen festgelegt sind", geschützt sind ("d'assurer la protection des droits de la femme ... tels que stipulés dans les déclarations et conventions internationales / to protect the rights of the woman ... as stipulated in international declarations and conventions"). Im Mittelpunkt der hiesigen Untersuchung stehen diese beiden Formulierungen, die das Spannungsfeld aus afrikanischen Traditionen und Wertvorstellungen einerseits und der Integration internationaler Vorschriften in die Charta andererseits zum Ausdruck bringen. Die möglichen Schwierigkeiten, die sich aus dieser Betonung der eigenen Werte und Tradition für den Schutz der Familie und für die Rechte der Frau ergeben können, werden nachfolgend beleuchtet und hierfür die Lösungen aufgezeigt, die die Afrikanische Charta bietet.

122; zum Protokoll Krisch, The Establishment of an African Court on Human and Peoples' Rights, Zeitschrift für ausländisches öffentliches Recht und Völkerrecht 58 (1998), S. 713 ff. Das Individuum wird hiernach vor dem Gerichtshof aber keine generelle Klagemöglichkeit erhalten; entsprechend der interamerikanischen und der früheren europäischen Regelung sollen in erster Linie die AfrKMRV und die Staaten zur Klageerhebung befugt sein, nachdem das Kommissionsverfahren durchlaufen ist. Zu den drei Verfahren und den Besonderheiten des bisherigen afrikanischen Systems Wittinger, Die drei regionalen Menschenrechtssysteme, Juristische Ausbildung 21 (1999), 405 ff. (409 ff.); zum interamerikanischen System Rensmann, Menschenrechtsschutz im Inter-Amerikanische-System: Modell für Europa?, Verfassung und Recht in Übersee 33 (2000), S. $137 \mathrm{ff}$.

Abschnitt 5 ("tradition and the values of African civilization") und 11 ("the importance traditionally attached") der Präambel; Art. 17 Abs. 3 ("morals and traditional values"); Art. 29 Abs. 7 AfrC ("positive African cultural values"); zu Art. 27 bis 29 AfrC und dem Charakteristikum der AfrC, Rechte mit Pflichten zu verknüpfen Wittinger, Familien und Frauen im regionalen Menschenrechtsschutz, Saarbrücker Studien zum internationalen Recht, Bd. 12 (Baden-Baden 1999), S. 306 ff. (319 f.), m.w.N.

Abschnitt 4 der Präambel, Art. 60 und Art. 62 AfrC. 


\section{Der Familienschutz in der Afrikanischen Charta: Die Familie als "Bewahrerin der in der Gemeinschaft anerkannten Sittlichkeit und der traditionellen Werte"}

Art. 18 AfrC widmet der Familie ${ }^{9}$ zwei Absätze. Art. 18 Abs. 1 Satz 1 AfrC enthält die Wertentscheidung, dass die Familie die "natürliche Einheit und Basis der Gesellschaft" ist. Im Unterschied zu Art. 17 Abs. 1 AMRK, zu Art. 23 Abs. 1 des Internationalen Pakts über bürgerliche und politische Rechte ${ }^{10}$ oder zu Art. 16 Abs. 3 der Allgemeinen Erklärung der Menschenrechte ${ }^{11}$ ist damit aber keine allgemeinen Schutzpflicht ("und hat ein Recht auf Schutz") verbunden. Art. 18 Abs. 1 Satz 2 AfrC statuiert die Pflicht des Staates in einem spezifischen Bereich, der Gesundheitsfürsorge, konkrete Maßnahmen zu ergreifen. ${ }^{12}$ Eine umfassende Unterstützungsverpflichtung statuiert Art. 18 Abs. 2 AfrC. ${ }^{13}$ Diese umfassende leistungsrechtliche Verpflichtung des Staates ist verknüpft mit der Rolle, die die Afrikanische Charta der Familie zuschreibt und beschränkt sich nach dem Wortlaut ("la famille dans sa mission de gardienne / the family, which is the custodian" ${ }^{14}$ ) auf diese Funktion

Zum Familienbegriff und zum Inhalt des Familienschutzes (Institutsgarantie, Leistungspflichten, Abwehrrecht) im Einzelnen Wittinger (Fn. 7), S. 62 ff.

10

Im folgenden: IPbürgR, v. 19.12.1966, abgedruckt in Simma / Fastenrath, Menschenrechte - Ihr internationaler Schutz (München, 3. Aufl. 1992), S. 22 ff.

11 Im folgenden: AllgErklMR, v. 10.12.1948, abgedruckt in: Simma / Fastenrath (Fn. 10), S. 5 ff.

12

Hierzu zählen z.B. medizinische Versorgung, die Verbesserung hygienischer Verhältnisse, gesundheitliche Aufklärung und Gesundheitsprogramme (vgl. hierzu die extensive Auslegung des Art. 16 AfrC, Schutz der Gesundheit, durch die AfrKMRV in Communication 25/89, Opinion of 4 April 1996 (19th Ordinary Session)/10 July 1996 (AHG/Res. 250 (XXXII), Free Legal Assistance Group et al. gegen Zaire, in Human Rights Law Journal 18 (1997), S. 32 f., Ziff. 47). Die daneben in Art. 18 AfrC enthaltene Pflicht, für die "seelische" Gesundheit der Familie Sorge zu tragen, bleibt demgegenüber offen und könnte auch die Gefahr des Eingriffs in das Familienleben durch den Staat zum "psychischen" Schutz der Familie bergen, dazu Elmadmad, in: Benedek / Heinz, Regional Systems of Human Rights Protection in Africa, America and Europe, Third African-American-European Conference 1992: Contributions to the Programme, Friedrich Naumann Stiftung (Brüssel 1992), S. 80.

Hierunter können die Pflicht zur Beschaffung von Nahrung, Kleidung und Unterkunft, eine allgemeine Verbesserung der Lebensbedingungen und finanzielle Hilfen für Familien fallen, ebenso medizinische Maßnahmen zum Schutz von Mutter und Kind, das Zurverfügungstellen von Wohnraum oder die Sicherung von Kinderbetreuung, ferner gesetzgeberische Maßnahmen, z.B. im Familien-, Sozial- und Steuerrecht, aber auch administrative oder praktische soziale Maßnahmen, vgl. hierzu die Berichtssystemsrichtlinien der AfrKMRV (Art. 62 AfrC) in Bezug auf Maßnahmen zum Schutz der Familie: Guidelines for national Periodic Reports, II. General Guidelines regarding the form and contents of reports on economic and social rights, (Guidelines for reporting on all the family; adequate standard of life and to the highest attainable level of health - Articles 16 and 18), Ziff. 28 ff.; Annex XII zum 2. Tätigkeitsbericht v. 14.6.1989, abgedruckt in: Human Rights Law Journal 11 (1990), S. 405 ff. (411 f.).

Hervorhebungen nicht im Original. 
der Familie als "Bewahrerin der in der Gemeinschaft anerkannten Sittlichkeit und der traditionellen Werte...".

Die Betonung der Familie als "Bewahrerin der in der Gemeinschaft anerkannten Sittlichkeit und traditionellen Werte" steht im Kontext mit dem Willen, dem befürchteten Verlust afrikanischer Werte, von Traditionen und Identität, der mit der Kolonialisierung einherging, aber auch durch Entwicklung und Fortschritt verursacht werden kann, entgegenzuwirken. Art. 18 Abs. 2 AfrC verkörpert daher besonders ausgeprägt die "gewisse konservative Urangst gegenüber gesellschaftspolitischen Veränderungen" ${ }^{16}$, denen die Familie als soziales Gebilde ausgesetzt ist. Kéba $M^{\prime} B a y e^{17}$ weist darauf hin, dass die Formulierung "la famille dans mission de gardienne de la morale..." im Zusammenhang mit der Einschätzung steht, dass die Familie traditionell eine spezifische Rolle bei der Erziehung der Jugend spielt, für deren "santé morale" sie Verantwortung trage und dass diese Wendung des Art. 18 Abs. 2 AfrC vor diesem Hintergrund erklärbar sei. Möglicherweise wurde die Formulierung mit der Intention aufgenommen, afrikanisches, als unproblematisch beurteiltes, familiäres Selbstverständnis zu verankern. Auch wenn manche afrikanische Autoren diese Bestimmung als unkritisch ansehen ${ }^{18}$, ergibt sich im übrigen kein geschlossenes Gesamtbild, das den Schluss zuließe, die Formulierung mute nur für Europäer ungewöhnlich an. ${ }^{19}$

Es ist offen, wann eine Familie die Anforderung, "Bewahrerin der Sittlichkeit und der in der Gemeinschaft anerkannten traditionellen Werte" zu sein, erfüllt, und wer über das Vorhandensein dieser Qualifikation entscheidet. Ist dies nur die Familie, die im Einklang mit Sitten und Gebräuchen lebt, z.B. die Polygamie praktiziert, kein freies Einverständnis der Ehepartner zur Heirat fordert und an ihren Töchtern Geschlechtsverstümmelungen vornehmen lässt? Oder ist es die Familie, die sich lediglich an die "guten" Traditionen hält,

Siehe auch: Kunig, in: Konrad (Hrsg.), Grundrechtsschutz und Verwaltungsverfahren unter besonderer Berücksichtigung des Asylrechts, Internationaler Menschenrechtsschutz, Schriften zum öffentlichen Recht Bd. 484 (Berlin 1985), S. 263; Benedek, The role of International Law in the protection and Promotion of Human Rights of Woman in Africa, Austrian Journal of Public International Law 49 (1995), S. 282. Nowak, UNO-Pakt über bürgerliche und politische Rechte und Fakultativprotokoll, CCPR-Kommentar (Kehl et al. 1989), Art. 23, Rn. 5.

Siehe z.B. M'Baye (Fn. 17), S. 182.

19 Siehe: Degni Segui, in: Benedek / Heinz, Regional Systems of Human Rights Protection in Africa, America and Europe Third African-American-European Conference 1992: Contributions to the Programme, Friedrich Naumann Stiftung (Brüssel 1992): Contributions to the Programme, S. 64; Elmadmad (Fn. 12), S. 79; Nhlapo, International protection of Human Rights and the Family: African Variations on a Common Theme, International Journal of Law and Family 3 (1989), S. 13. 
z.B. den Respekt und die Betreuung der älteren Familienmitglieder? ${ }^{20}$ Ist dies nur die traditionelle Großfamilie? Was bedeuten Sittlichkeit und welche Traditionen gelten als anerkannt? Der unbestimmte, in der Charta nicht näher präzisierte Begriff der "Sittlichkeit" kommt im übrigen nicht nur in der Afrikanischen Charta vor, sondern auch in den beiden Konventionen $^{21}$, allerdings nicht in Zusammenhang mit der Funktion der Familie; gleiches gilt für die "Moral", wie "la morale / the morals" auch übersetzt werden könnte. Die Europäischen Organe haben insoweit festgehalten, dass kein einheitlicher europäischer Moralbegriff besteht und den Staaten insoweit ein Ermessensspielraum zusteht ${ }^{22}$; dies könnte auch auf die afrikanischen Staaten übertragen werden, die dann diesen Begriff ausfüllen könnten. Mit der Festlegung dieser Rolle der Familie durch die Charta kann eine Beschränkung des Schutzes auf bestimmte, "adäquate" Familien einhergehen oder auf bestimmte Familienformen, die nicht der erweiterten, traditionellen afrikanischen Familie - welche in erster Linie mit der Erfüllung dieser sozialen Funktion verbunden werden mag - entsprechen (z.B. alleinerziehende Eltern, unverheiratete Eltern). Diese könnten daher möglicherweise von dem Leistungsschutz des Art. 18 Abs. 2 AfrC ausgeschlossen werden. Für die berechtigte Kopplung des leistungsrechtlichen Familienschutzes an die Rolle der Familie als Wächterin über "Sittlichkeit und traditionelle Werte" könnte andererseits ein legitimes Interesse des Staates sprechen, mit seinen Ressourcen nur solche Familien aktiv zu unterstützen, die er für "förderungswürdig" hält.

Selbst wenn man Art. 18 Abs. 2 AfrC in diesem einschränkenden Sinne verstehen will, bedeutet dies aber nicht einen völligen Ausschluss anderer Familien (-formen) von der Pflicht des Staates zu leistungsrechtlichem Schutz: In Bezug auf den Gesundheitsbereich kann eine Schutzpflicht aus Art. 18 Abs. 1 Satz 2 AfrC resultieren, der anders als Art. 18 Abs. 2 AfrC keinen Zusatz über die Funktion der Familie als Hüterin von "Sittlichkeit und Traditionen" enthält. Nur wenn Art. 18 Abs. 2 AfrC als Präzisierung oder als lex specialis zu Abs. 1 in Bezug auf die sich hieraus ergebenden positiven und leistungsrechtlichen Verpflichtungen zu werten wäre, könnte ein Rückgriff nicht stattfinden. Dies ist offen. Einerseits könnte Art. 18 Abs. 2 AfrC als Konkretisierung in leistungsrechtlicher Hinsicht und als Bindung an die Familie als "Wächterin über Sitte und Werte" verstanden werden. Andererseits spricht die vorab geregelte spezielle Gesundheitsfürsorgepflicht für eine

Zu dem Prinzip der Solidarität: Mbaya, E., La charte africaine en tant que méchanisme de protection des droits de l'homme, in: Bernhardt / Jolowicz (Hrsg.), International Enforcement of Human Rights, Beiträge zum ausländischen öffentlichen Recht Bd. 93 (Berlin et al. 1987), S. 96; Rwezaura, Tanzania: Family Law and the New Bill of Rights, Journal of Family Law 29 (199091), S. 455.

21 So: Art. 6 Abs. 1, Satz 2, Art. 8 Abs. 2 - 11 Abs. 2 EMRK; Art. 12 Abs. 3, Art. 13 Abs. 2 b) AMRK. 
abschließende Regelung auf diesem Gebiet, ohne eine bestimmte Qualifizierung der Familie zu fordern.

$\mathrm{Zu}$ einer Lösung, mit der ein umfassender - nicht nur auf die Gesundheitsfürsorge begrenzter - leistungsrechtlicher Schutz der Familie erreicht wird, der nicht auf ihre Qualifizierung als Bewahrerin der "Sittlichkeit und der Werte" beschränkt ist, führt Art. 60 AfrC. Er enthält die Rechtsgrundlagen, von denen sich die Afrikanische Menschenrechtskommission "leiten lassen" ("s'inspire / draw inspiration") soll: die internationalen und afrikanischen Menschenrechtsinstrumente. Art. 60 AfrC mit seiner Betonung der universellen Menschenrechtschutzinstrumente, namentlich der AllgErklMR, ist Beweis für die Einbettung der Afrikanischen Charta in den internationalen Menschenrechtsschutz. Trotz der Unterstreichung der afrikanischen Identität und Tradition in der Charta, wie sie in Art. 18 Abs. 2 AfrC zum Ausdruck kommt, will die Afrikanische Charta nicht außerhalb dieses Systems stehen. Art. 60 AfrC, auf den im Zusammenhang mit den Rechten der Frau noch zurückzukommen sein wird, wirft allerdings verschiedene Fragen auf: Zum einen ist nicht klar, wie der Begriff des "leiten Lassens" zu verstehen ist. Zutreffenderweise wurde darauf hingewiesen, dass diese Formulierung weniger der juristischen als einer "poetischen" Sprache entspricht und auslegungsfähig ist. ${ }^{23}$ " $(S)^{\prime}$ inspire / draw inspiration" kann bedeuten, dass sich die Afrikanische Kommission bzw. der künftige Gerichtshof nur an internationales Recht "erinnern" soll, ohne daran gebunden zu sein oder aber, dass universelle Menschenrechtsnormen bei der Beurteilung von Beschwerden und der Interpretation von einzelnen Chartavorschriften konkret anwendbar sind, in dem Sinne, dass die Vereinbarkeit der Chartavorschriften mit internationalem Recht verlangt wird. ${ }^{24}$ Für einen solchen bindenden Interpretationsgrundsatz spricht die Überschrift des Kapitels, dessen erste Vorschrift Art. 60 AfrC ist und die lautet: "Anwendbare Grundsätze" ("Des Principes Applicables / Applicable Principles"). ${ }^{25}$ Die Auslegung der Chartavorschriften soll damit nach der Intention des Art. 60 AfrC nicht hinter dem internationalen Standard zurückbleiben. Die Bejahung einer solchen Vereinbarkeit schließt es aus, dass die Charta von den Organen entgegen internationalen Prinzipien ausgelegt wird. Insbesondere kommen Auslegung und Umfang von internationalen Vorschriften zum Zuge, wenn eine Chartavorschrift ausfüllungsbedürftige Begriffe enthält, wie z.B. die "Sittlichkeit und die anerkannten traditionellen Werte" in Art. 18 Abs. 2 AfrC.

23

24

25

Hierzu und zum folgenden Degni Segui (Fn. 19), S. 62.

Siehe: Buergenthal / Kiss, La protection Internationale des Droits de l'Homme, Précis (Kehl et al. 1991, S. 137; Okere, The protection of Human Rights in Africa and the African Convention on Human and Peoples' Rights: A comparative Analysis with the European and the American System, Human Rights Law Quarterly 6 (1984), S. 149; Wean, Real protection for African Woman? The African Charter on Human and Peoples' Rights, Emory Journal of international dispute resolution 2 (1988), S. 432 und 446.

Im Original keine Hervorhebung. 
Die Hürde der Vereinbarkeit von Chartabestimmungen mit den Prinzipien des internationalen Rechts bedeutet, dass z.B. über die Heranziehung von Art. 23 IPbürgR und Art. 16 Abs. 3 AllgErklMR eine Ausgrenzung bestimmter Familien oder Familienformen ausscheidet. Denn diese Bestimmungen enthalten in ihren Gewährleistungspflichten, die ihren Institutsgarantien immanent sind, keine Zusätze über eine bestimmte Qualifizierung der Familie. Da die Allg ErklMR in Art. 60 AfrC ausdrücklich als Instrument aufgeführt ist, das die Afrikanische Kommission anwenden soll, stellt sich im übrigen nicht die Problematik ihrer Rechtsverbindlichkeit. ${ }^{26}$ Art. 60 AfrC enthält mit seiner Formulierung der "anderen, von den Vereinten Nationen und von afrikanischen Staaten angenommenen Instrumenten" aber eine Unsicherheit, die zu einer wesentlichen Einschränkung führen kann, wenn hieraus das Erfordernis einer formalen Mitgliedschaft des im Beschwerdeverfahren angegangenen Staates gefolgert wird. Art. 60 AfrC scheint insgesamt, wie auch in seinem letzten Passus, der von den "Instrumenten der Sonderorganisationen der Vereinten Nationen, bei denen die Vertragsstaaten der Charta Mitglieder sind" spricht, eine Vertragsmitgliedschaft zu fordern. Ein Vergleich mit Art. 61 AfrC ("allgemeine oder besondere internationale Abkommen, die Regeln enthalten, die ausdrücklich von Mitgliedstaaten der Organisation für Afrikanische Einheit anerkannt worden sind") als der zweiten Vorschrift, die die von der Kommission zu berücksichtigenden Prinzipien enthält, stützt dieses Ergebnis, da auch hierin ein Bekenntnis zur Verbindlichkeit von internationalem Recht nur bei Zustimmung der Staaten gesehen werden kann. ${ }^{27}$ Selbst bei Annahme einer erforderlichen Mitgliedschaft nach Art. 60 AfrC ist aber mit Blick auf Art. 23 IPbürR darauf hinzuweisen, dass die Mehrzahl der afrikanischen Staaten Vertragsstaaten dieses Internationalen Paktes sind. ${ }^{28}$ Wird eine Vertragsmitgliedschaft vorausgesetzt, bringt dies zugleich die Beachtlichkeit von Vorbehalten, die die Staaten zu Familienschutzvorschriften internationaler Instrumente erklärt haben, mit sich. ${ }^{29}$ Dies bedeutete eine erhebliche Einschränkung des Art. 60 AfrC.

Fraglich ist bei der Auslegung des Art. 60 AfrC ferner, ob auch Vorschriften der beiden regionalen Menschenrechtskonventionen hierüber anwendbar sind. Rein formal betrachtet, könnten die regionalen Konventionen, die bei Schaffung der Charta bekannt waren und auch als Vorbild galten, von denen man sich aber unter Betonung der afrikanischen Identität auch abgrenzen wollte, als ausgespart gelten, da sie nicht explizit aufgeführt sind. Ande-

Zur Verbindlichkeit der AllgErklMR nur Stern, Das Staatsrecht der Bundesrepublik Deutschland, Allgemeine Lehren der Grundrechte, Bd. III/2 (München 1994), S. 1529.

Dazu Umozurike, The African Charter on Human and Peoples' Rights, American Journal of International Law 77 (1983), S. 911.

Zur Mitgliedschaft afrikanischer Staaten die Übersicht bei Marie, HRLJ 20 (1999), S. 120 f.

29

$\mathrm{Zu}$ den Vorbehalten afrik. Staaten zum UN-Übereinkommen zur Beseitigung jeder Diskriminierung der Frau (im Folgenden: CEDAW) v. 18.12.1979 (in: Simma / Fastenrath (Fn. 10), S. 194 ff.), Elmadmad (Fn. 12), S. 82. 
rerseits werden die beiden regionalen Instrumente üblicherweise als Teil des "internationalen Rechts" eingeordnet, die zusammen mit den universellen Instrumenten das internationale Recht im Bereich der Menschenrechte ausmachen und aufgrund der gegenseitigen Beeinflussung nicht als Gegensatzpaar zu begreifen sind. In der Literatur wird daher trotz des Bemühens, bei Schaffung der Charta nicht en bloc Vorschriften aus der EMRK zu übernehmen, zu Recht gefolgert, dass in Art. 60 AfrC ("international law") und in Art. 61 AfrC ("legal precedents") die beiden regionalen Konventionen eingeschlossen sind. ${ }^{30}$ Damit ist auch Art. 17 Abs. 1 AMRK, der keine Eingrenzung auf bestimmte Familienformen enthält, für die Auslegung des Art. 18 Abs. 2 AfrC heranzuziehen.

Problematisch an diesem Rückgriff auf internationales und als dessen Bestandteil auch auf regionales Recht über Art. $60 \mathrm{AfrC}$ ist allerdings, dass mit der Proklamation des Stellenwertes der Familie als "Bewahrerin der in der Gemeinschaft anerkannten Sittlichkeit und traditionellen Werte" möglicherweise gerade ein "afrikanisches Spezifikum" geschaffen werden sollte - in Unterscheidung $\mathrm{zu}$ entsprechenden internationalen oder regionalen Regelungen - das über das internationale Recht "umgangen" würde. Durch die Akzeptanz des Art. 60 AfrC haben die Mitgliedstaaten jedoch zugleich die Einbettung in internationale Vorschriften angenommen. Abgesehen von dem leistungsrechtlichen Schutz für alle Familienformen eröffnet Art. 60 AfrC darüber hinaus die Möglichkeit, zu einer abwehrrechtlichen Komponente des Familienschutzes in Art. 18 Abs. 1 und 2 AfrC zu kommen. Hierbei ist allerdings zu berücksichtigen, dass damit über die dortigen wirtschaftlichen Leistungsrechte hinausgegangen wird. Durch die Heranziehung einer echten Institutsgarantie als umfassender Schutzgarantie aus dem internationalen oder regionalen Recht wie Art. 17 Abs. 1 AMRK, kann ferner die "unvollkommene" Garantie in Art. 18 Abs. 1 Satz 1 AfrC durch Interpretation entsprechend ausgedehnt werden.

Zusammenfassend lässt sich damit festhalten, dass die Wendung "Bewahrerein der Sittlichkeit und der traditionellen Werte" über das internationale Recht als Interpretationshilfe relativiert werden kann, so dass "missliebige" Familien oder Familien, die beispielweise nicht der traditionellen Großfamilienstruktur entsprechen, nicht vom Familienschutz der Charta ausgeschlossen werden.

30

Sieghart, The International Law of Human Rights (Oxford 1983), S. 418; Bello, The African Charter on Human and Peoples' Rights, A Legal Analysis, Académie de droit international de la Haye, Recueil des Cours 194 (1985 V), S. 82 ff. (86 f.); auch Benedek, Durchsetzung von Rechten des Menschen und der Völker in Afrika auf regionaler und nationaler Ebene, Zeitschrift für ausländisches öffentliches Recht und Völkerrecht 54/1 (1994), S. 178 (Anm. 89) zur Empfehlung der AfrKMRV, die AfrC auch im Sinne regionaler Instrumente zu interpretieren. 


\section{Die Position der Frau in der Afrikanischen Charta}

\section{Die Rechte der Frau und Art. 18 Abs. 1 und 2 AfrC: Schutz der Familie versus Schutz des Individuums?}

Rechtsträger des Art. 18 Abs. 1 und 2 AfrC ist die Familie als solche, als Gruppe und Kollektiv. Ein ausdrückliches Individualrecht auf Schutz des Familienlebens, wie ihn etwa Art. 8 EMRK und Art. 11 Abs. 2 AMRK (neben Art. 17 Abs. 1 AMRK) garantieren, enthält die Charta dagegen nicht. Dieser Befund führt in der Afrikanischen Charta zu der Problematik, dass sich der Einzelne im Falle eines möglichen Konfliktfalles zwischen Individuum und Familie, z.B. beim Scheitern einer Ehe, nicht auf eigene Familienschutzvorschriften der Charta stuitzen und keine positive Verpflichtung des Staates zum Eingreifen, z.B. mittels des Familienrechts, einfordern kann. Anders als in den beiden Konventionen gibt es darüber hinaus auch keine Festschreibung von Ehevorschriften, wie etwa einen Gleichheitssatz der Eheleute (Art. 5 des 7. ZP EMRK, Art. 17 Abs. 4 AMRK), der hier eingreifen könnte. Ausdrücklich schützt die Charta nur die Familie als Einheit. Eine gegensätzliche Interessenlage wurde angesichts der afrikanischen traditionellen Verwebung zwischen dem Einzelnen und der Gruppe / Familie ${ }^{31}$ möglicherweise nicht erkannt oder als nicht regelungsbedürftig erachtet. Art. 18 Abs. 1 und 2 AfrC ist damit Ausdruck des traditionell ausgeprägten Familienbezuges der Afrikaner und macht die "Einbindung des Einzelnen" ${ }^{32}$ in die Familie deutlich. Diese Integrierung birgt Gefahren, speziell für weibliche Familienmitglieder, deren familiäre Position traditionell der des Haushaltsvorstandes und Ehemannes untergeordnet ist. ${ }^{33}$ Eine Beschwerdeführerin könnte sich damit anders als z.B. im europäi-

Krit. zum Spannungsverhältnis zwischen dem Individuum und der traditionellen afrikanischen Gruppenorientiertheit z.B.: Howard, Human Rights in Commonwealth Africa (New Jersey 1986), S. 19 f.; Eze, Human Rights Issues and Violations: the African Experience, in: Sheperd / Anikpo (Hrsg.), Emerging Human Rights, The African political Economy Context (Westport 1990), S. 90; abl. Zur Übertragung des Konzeptes "Individuum versus Gruppe" als in Afrika unbekannter Konflikt, der westlichen/europäischen Ursprungs sei z.B. Legesse, Human Rights in African Political Culture, in: Thompson (Hrsg.), The Moral Imperatives of Human Rights: A World Survey (Washington 1980), S. $124 \mathrm{f}$.

So ohne Problematisierung Palm-Risse, Der völkerrechtliche Schutz von Ehe und Familie (Berlin 1990), S. 69.

33

Ausführlich zur rechtlichen Situation von Frauen in der traditionellen afrikanischen Gesellschaft Dagui, Revue Juridique et Politique 45 (1991), S. 196 ff. (199 f.); siehe z.B. auch die Regelungen afrikanischer Staaten über den Ehemann als Familienoberhaupt, z.B. in: Somalia, Art. 4 (2) Personalstatutgesetz v. 11.1.1975, in: Bergmann / Ferid, Internationales Ehe- und Kindschaftsrecht (Frankfurt 1997, Loseblatt), Somalia, S. 10; der Elfenbeinküste, Art. 58 Gesetz Nr. 64-375 v. 7.10.1964 über die Ehe, in: Bergmann / Ferid, ebenda, Elfenbeinküste, S. 25 (auch keine Änderung durch die als "Gesetzesrevolution" bezeichnete Neuerungen des ivorianischen Ehe- und Familienrechts, Bergmann / Ferid, ebenda, S. 10). 
schen Fall Airey gegen Irland (1979) ${ }^{34}$ vor den Afrikanischen Organen nicht mit Hilfe des Art. 18 Abs. 1, 2 AfrC auf den Schutz ihres Privat- und Familienlebens stützen, um die prozessuale Durchsetzung einer ehelichen Trennung zu erreichen, sofern eine solche Möglichkeit innerstaatlich vorgesehen ist.

Als Lösung dieses Defizits des fehlenden Individualschutzes wird in der Literatur mitunter versucht, Art. 18 Abs. 1 und Abs. 2 AfrC in der Weise zu interpretieren, dass die individuellen Rechte der einzelnen Familienmitglieder, und insbesondere die von Frauen, neben den Rechten der Familie als Kollektiv existieren. ${ }^{35}$ Danach regulierten die individuellen Rechte die innerfamiliären Beziehungen und bildeten die Basis für die Familie als kollektive Einheit. Um diese Gemeinschaft zu bewahren, sollten Individualrechte grundsätzlich im Sinne der Interessen des Kollektivs ausgeübt werden. Im Konfliktfall jedoch könnten individuelle Rechte gegenüber der Familie oder anderen Familienmitglieder durchgesetzt werden. Kritisch an dieser Interpretation ist, dass der Wortlaut und Sinn des Art. 18 Abs. 1, 2 AfrC diese kaum stützen. Die Familie wird hier als Institution und soziale Einheit betrachtet, die eine bestimmte Rolle, die der "Bewahrerin von Sittlichkeit und traditionellen Werten", zu erfüllen hat. Die klassische Lesart solcher familiärer Institutsgarantien, die den Staat verpflichtet, zur Erhaltung der Familie beizutragen oder negativen Folgen ihrer Auflösung entgegenzutreten, stehen einer solchen individualrechtlich ausgerichteten Auslegung entgegen, auch wenn diese wünschenswert wäre; ganz abgesehen davon, dass offen ist, ob die Afrikanischen Organe einer solchen Interpretation des Art. 18 Abs. 1, 2 AfrC folgen würden. Denn formal schützt Art. 18 Abs. 1, 2 AfrC die Rechte der Familie, nicht familiäre Rechte des Einzelnen.

Eine weitere Möglichkeit, einen Individualschutz zu erreichen, könnte in Art. 60 AfrC liegen. Es ist fraglich, ob die Integration solcher Vorschriften, die in der Charta überhaupt nicht vorhanden sind, über Art. 60 AfrC bewerkstelligt werden kann. ${ }^{36}$ Wie dargelegt, ist Art. 60 AfrC im Sinne einer Vereinbarkeit der Chartavorschriften mit internationalem Recht durch entsprechende Interpretation unklarer oder auslegungsfähiger Vorschriften zu verstehen. Die Schließung von Lücken der Charta mit Hilfe des internationalen Rechts bedeutet demgegenüber noch einen weiteren Schritt. Festzuhalten ist, dass Art. 60 AfrC keine Eingrenzung nur auf unklare Vorschriften enthält und lediglich festlegt, dass sich die

34

35

36

Urt. v. 9.1.1979, Série A Nr. 32 = EuGRZ 6 (1979), S. 626 ff.

Beyani, Towards a more effective Guarantee of Womens' Rights in the African Human Rights System, in: Cook (Hrsg.), Human Rights of Woman (Philadelphia 1994), S. 291 f.; Armstrong / Beyani / Himonga / Kabeberi-Macharia / Molokomme / Ncube / Nhalpo / Rwezaura / Stewart, Uncovering Reality: Excavating Women's Rights in African Family Law, International Journal of Law and Family 7 (1993), S. 321

Bejahend Tonndorf, Menschenrechte in Afrika, Konzeption, Verletzung und Rechtsschutz im Rahmen der OAU Freiburger Beiträge zur Entwicklung und Politik, Bd. 24 (Freiburg 1997), S. 287 u. 376 f.; a.A. wohl Degni Segui (Fn. 19), S. 62 
Afrikanische Menschenrechtskommission von internationalem Recht leiten lassen soll, ohne dies näher zu präzisieren. Für eine solche Ansicht, über Art. 60 AfrC (" $s^{\prime}$ inspire / draw inspiration") nicht nur eine Interpretation und Vereinbarkeit vorhandener Chartavorschriften mit internationalen Menschenrechtsnormen zu verlangen, sondern hierüber die Einbeziehung nicht geregelter Gebiete in die Charta zuzulassen, spricht daher die weite Fassung des Art. 60 AfrC. Dem Einwand eines Eingriffes in die Staatsautonomie könnte die freiwillige Chartamitgliedschaft und die Akzeptanz einer solchen Ausdehnungsmöglichkeit durch die Mitgliedstaaten entgegengehalten werden. Andererseits könnten somit wie bei der Überwindung der Spezifizierung der Familie als "Bewahrerin der Sittlichkeit und der traditionellen Werte" - Lücken geschlossen werden, die möglicherweise bewusst in Kauf genommen wurden, unter Setzung bestimmter "afrikanischer Akzente", wie der Nachrangigkeit eines Privat- und Familienlebens.

\section{Die Rechte der Frau und die Familie als "Bewahrerin der Traditionen"}

Der fehlende explizite Individualschutz im Falle eines Konfliktes mit der Familie verschärft sich durch die oben bereits untersuchte Qualifikation der Familie als "Bewahrerin der traditionellen Werte", da gerade Frauen von den "traditionellen Werten" und deren rechtlicher Verfestigung in Gewohnheits- und Stammesrecht betroffen sind, das ihre juristische Schlechterstellung zementiert. ${ }^{37} \mathrm{Zu}$ nennen sind insbesondere gewohnheitsrechtliche Regelungen im Familien- und Erbrecht, z.B. fehlende Rechte der Frau im Zusammenhang mit der Ehe, wie ein fehlendes Mindestheiratsalter, ihre nicht erforderliche freie Zustimmung zur Ehe, Scheidungsverbote oder Verlust von Sorgerechten, ferner die eigentums- und erbrechtliche Schlechterstellung von Frauen, die sich in dem gewohnheitsrechtlichen Ausschluss, Clan-Land weder erwerben noch vererben zu können, manifestiert. Auch der Status unverheirateter Frauen könnte unter dem Schutzmantel dieser Begriffe verletzt werden angesichts der traditionell vorkommenden Ausstoßung dieser Frauen aus der Gemeinschaft, getragen von der Sorge, die Gemeinschaft zu schützen. ${ }^{38}$ Hinzukommen nicht zuletzt Traditionen wie die Geschlechtsverstümmelung an Frauen. ${ }^{39}$ 
Die für die Positionen von Frauen, auch innerhalb und gegenüber der Familie, entscheidende $^{40}$ Vorschrift ist Art. 18 Abs. 3 AfrC, der zwei Komponenten umfasst:

Zum einen wird die Pflicht des Staates aufgeführt, für die Beseitigung der Diskriminierung von Frauen zu sorgen, also die explizite positive Verpflichtung zum Einschreiten statuiert, um de jure oder de facto bestehende Diskriminierungen zu unterbinden. Die Ergreifung von Maßnahmen zum Ausgleich traditioneller Schlechterstellungen und die Pflicht zu Förderungsmaßnahmen von Frauen ist Teil dieser Verpflichtung, da "jede" ("every / toute") Diskriminierung gegenüber Frauen beseitigt werden soll. Art. 18 Abs. 3 AfrC hat mit seinem speziellen Diskriminierungsverbot für Frauen das Prinzip des internationalen Menschenrechtsschutzes aufgenommen, die herkömmliche Benachteiligung bestimmter Gruppen durch positive Maßnahmen auszugleichen. ${ }^{41}$ Für die Position der Frau als Individuum gegenüber der Familie ist von Bedeutung, dass dieses spezielle Diskriminierungsverbot Frauen auch vor Diskriminierung innerhalb der Familie schützt. Denn das spezielle Diskriminierungsverbot des Art. 18 Abs. 3 AfrC, das von dem Staat ohne Einschränkung die Erfüllung der Beseitigung von Diskriminierung fordert, entfaltet auch eine horizontale Wirkung im privaten Bereich. Eine Vorrangigkeit etwaiger kollektiver Rechte der Familie oder einzelner Familienmitglieder ist damit nicht vereinbar. Diskriminierende Regelungen z.B. zugunsten des Ehemannes in Bezug auf die ehelichen Vermögensverhältnisse oder Sorgerechte für Kinder verstoßen gegen Art. 18 Abs. 3 AfrC. Ebenso ist das Diskriminierungsverbot das Regulativ für die Auslegung der Begriffe der "Sittlichkeit und traditionellen Werte".

Zum anderen muss der Staat nach Art. 18 Abs. 3 AfrC die "Rechte der Frau, wie sie in internationalen Deklarationen und Konventionen festgelegt sind", sicherstellen ("assurer / ensure"), also positiv, gesetzlich oder faktisch, einschreiten. Es fragt sich, was unter den "Rechten der Frau, wie sie in internationalen Deklarationen und Konventionen festgelegt sind", zu verstehen ist. Eine derartige Bezugnahme auf internationales Recht ist selten und

Siehe auch das Draft Protocol To The African Charter On Human And Peoples' Rights Concerning The Rights Of Women; Experts Meeting On Preparation Of A Draft Protocol To The African Charter On Human And Peoples' Rights Concerning The Rights Of Women, organised by The International Commission of Jurists in collaboration with The African Commission On Human And Peoples' Rights, 12-14-April 1997, Nouakchott / Islamic Republic of Mauretania und Report on the First Meeting of the Working Group on the Additional Protocol to the African Charter on Women's Rights, held from 26-28 January, in Banjul, Gambia, DOC/OS/34c (XXIII); Annex I: Amended Draft Protocol; hierzu insgesamt Wittinger (Fn. 7), S. 202 ff. 
existiert in dieser Form weder in der Europäischen noch in der Amerikanischen Menschenrechtskonvention. Dieser Teil der Vorschrift könnte einerseits als ein nur deklaratorisches Bekenntnis zum internationalen Recht verstanden oder aber als ein echter Verweis auf internationale Normen bewertet werden, der zu deren unmittelbarer Anwendbarkeit führt.

Nach dem Wortlaut ist letzteres der Fall. Der Hinweis auf internationales Recht, der nicht mit Einschränkungen versehen ist, eröffnet die Anwendbarkeit dieser internationalen Instrumente. Der Verweis bietet so die Möglichkeit, dass sich Frauen im Individualbeschwerdeverfahren auf Rechte aus allen internationalen Instrumenten berufen können. ${ }^{42}$ Eine besondere Ausweitung für die Rechte von Frauen bedeutet der Verweis, wenn keine Vertragsmitgliedschaft des Staates bezüglich des jeweiligen Instrumentes gefordert wird und damit auch die Vorbehalte zu internationalen Bestimmungen unbeachtlich sind. Es ist aber offen, ob dieser Rückgriff auf internationales Recht auch zur Anwendbarkeit einschlägiger Übereinkommen führt, bei denen die Mitgliedstaaten der Afrikanischen Charta keine Vertragsstaaten sind. ${ }^{43}$ Hiergegen spricht das allgemeine Prinzip, dass Staaten nicht zur Einhaltung von Pflichten angehalten werden können, die sie nicht eingegangen sind. Für den Verzicht auf eine Vertragsmitgliedschaft spricht hingegen entscheidend die fehlende Aufstellung einer solchen ausdrücklichen Prämisse, ferner der Formulierungsunterschied zwischen Art. 18 Abs. 3 AfrC und Art. 60 AfrC, der im Gegensatz zu Abs. 3 die Forderung einer Mitgliedschaft der Staaten zu den dort aufgeführten Instrumenten nahe legt. Für diese Konsequenz spricht ferner die freie Entscheidung der Mitgliedstaaten, die der Afrikanischen Charta beigetreten sind und die keine Einschränkungen zu der Vorschrift erklärt haben, wie auch insgesamt zur Charta keine Vorbehalte abgegeben wurden. Dieser Gesichtspunkt ist auch entscheidendes Argument dafür, den Verweis in Art. 18 Abs. 3 AfrC auf "internationale Deklarationen", denen üblicherweise kein bindender Vertragscharakter zukommt, als Pflicht der Mitgliedstaaten zu interpretieren, unverbindliche Instrumente mit nur "moralischer" Wirkung als bindendes Recht anzuerkennen.

Für die Interpretation des Verweises als echten "renvoi" streitet neben dem Wortlaut, der Sinn und Zweck der Vorschrift. Es ist nicht ersichtlich, weshalb ein solcher Verweis aufgenommen werden sollte, wenn er nicht die Erweiterung des Menschenrechtsschutzes zur Konsequenz haben sollte. Ein nur deklaratorischer Charakter des Verweises in Bezug auf das internationale Recht widerspricht der in der Vorschrift festgelegten Schutzverpflichtung des Staates, der die Verwirklichung dieser in "internationalen Erklärungen und Übereinkommen" geschützten Rechte "sicherstellen" ("assurer / ensure") muss. Ein bloßer Verweis ohne rechtliche Konsequenz hätte unterbleiben können, denn pauschale Bekenntnisse zum

Hierzu z.B. Ouguergouz, La charte africaine des droits de l'homme et des peuples (Genève / Paris 1993), S. $126 \mathrm{f}$.

43

Abl. Umozurike, The African Charter on Human and Peoples' Rights (The Hague et al. 1997), S. 57. 
internationalen Recht sind z.B. in der Präambel der Charta ${ }^{44}$ bereits vorhanden. Die Bezugnahme auf internationales Recht in Art. 18 Abs. 3 AfrC ist damit als Anerkennung der Einheit der internationalen Menschenrechtsschutzregeln zu sehen, aus der konkrete, juristische Folgen erwachsen. Hinzu kommt hier die Entstehungsgeschichte des Art. 18 Abs. 3 AfrC. Denn bei dem Zusatz der "Rechte der Frau" handelt es sich um eine erst nachträglich eingefügte Wendung, die im Entwurf der Charta noch fehlte. ${ }^{45}$ Dort erstreckte sich die Formulierung "wie sie in internationalen Deklarationen und Konventionen festgelegt sind" nur auf die ebenfalls in Art. 18 Abs. 3 geschützten Rechte der Kinder. Mit der Einbeziehung der Rechte der Frau handelt es sich also um eine gewollte Erweiterung gegenüber dem schwächeren Entwurf, der in Bezug auf Frauen lediglich das spezielle Diskriminierungsverbot vorsah. Damit liegt es nahe, dass die Staaten eine bewusste Ausdehnung des Schutzes erreichen wollten, zu der auch die konkrete Anwendbarkeit internationalen Rechts im Beschwerdeverfahren gehören sollte.

Nach der hier vertretenen Auslegung hat der Verweis auf das internationale Recht weitreichende Konsequenzen und erhöht die anwendbaren Bestimmungen zum Schutz von Frauen entscheidend: so werden z.B. neben absoluten ${ }^{46}$ und weiteren speziellen Diskriminierungsverboten $^{47}$ sowie Gleichheitsgeboten ${ }^{48}$, insbesondere Eheregelungen ${ }^{49}$ und Schutzvorschriften für Frauen in ihrer Position in der Familie ${ }^{50}$ oder im Arbeitsleben ${ }^{51}$ anwendbar. Somit wird über die Vorschrift des Art. 18 Abs. 3 AfrC im Ergebnis ebenso viel erreicht wie über spezielle Menschenrechtsverträge zum Schutz von Frauen, deren Inhalt über die Vorschrift integriert werden können. Auch regionale Instrumente, wie die beiden Menschenrechtskonventionen, die nicht als Antagonismus zum internationalen Recht, sondern als dessen Bestandteil zu beurteilen sind, können einbezogen werden.

Abschnitt 4 u. 10 der Präambel.

Siehe Art. 18 Abs. 3 AfrC-Draft, abgedruckt in: Kunig / Benedek / Mahalu, Regional Protection Of Human Rights By International Law: The Emerging African System, VRÜ Beiheft 12 (BadenBaden 1985), S. 112.

So Art. 26 IPbürgR.

So Art. 2, 3, 7 ff. CEDAW.

Siehe z.B. die Art. 3 des IPbürgR und des Internationalen Paktes über wirtschaftliche und soziale Rechte (IPwirtR).

Siehe: das Verbot der Zwangsheirat in Art. 1 c) (i), (ii), (iii) und die Festlegung des Mindestalters für eine Ehe in Art. 2 des Zusatzabkommens über die Abschaffung der Sklaverei, des Sklavenhandels und sklavereiähnlicher Praktiken v. 7.9.1956.

So Art. 16 e) CEDAW (Freiheit der Familienplanung), Art. 16. h) CEDAW (Eigentumsregeln in der Ehe).

Vgl. Art. 7 IPwirtR. 
Von hoher Relevanz ist der Verweis auf die "internationalen Konventionen und Deklarationen" gerade für solche Bestimmungen, die in der Charta nicht existieren, also außer den Ehevorschriften, insbesondere für individualrechtliche Ansprüche auf Schutz des Familienlebens, die so in die Charta integriert werden können. Die offene Frage, ob durch Art. 60 AfrC über die reine Interpretation vorhandener Chartavorschriften im Sinne des internationalen Rechts hinaus, auch nicht geregelte Bereiche einbezogen werden können, relativiert sich damit. Der Vergleich des Art. 60 AfrC mit Art. 18 Abs. 3 AfrC spricht im übrigen eher gegen die Bejahung einer solchen Einbeziehung fehlender Vorschriften im Rahmen des Art. 60 AfrC. Denn in Art. 18 Abs. 3 AfrC wird in einer konkreten Vorschrift an Rechte bestimmter Personen angeknüpft ("les droits de la femme / the rights of women"), während Art. 60 AfrC nur eine allgemeine Bezugnahme auf internationales Recht enthält. Es ist auch ins Gedächtnis zu rufen, dass bei Art. 60 AfrC im Unterschied zu Art. 18 Abs. 3 AfrC die Erforderlichkeit einer Mitgliedschaft des Staates zu dem in Frage stehenden Instrument nahe liegt und damit auch entsprechende Vorbehalte (z.B. zu Ehevorschriften) berücksichtigt werden müssten. Der in Art. 18 Abs. 3 AfrC enthaltene Verweis auf das internationale Recht wirkt sich beim Individualschutz in zweifacher Hinsicht aus. Er führt einerseits zur Geltung entsprechender Individualrechte zugunsten von Frauen, die das Familienleben des Einzelnen - auch im Verhältnis zu den übrigen Familienmitgliedern schützen. ${ }^{52}$ Andererseits ist er maßgebend für das Kriterium der Bewahrung von "Sittlichkeit und traditionellen Werten", mit der Folge dass Traditionen, moralische und kulturelle Werte, die die Familie zu schützen hat, danach mit den internationalen Menschenrechtsstandards, wie Gleichheitsgeboten und Diskriminierungsverboten vereinbar sein müssen. Eine Auslegung des Art. 18 AfrC, die es zulässt, dass das Familienoberhaupt oder der Staat entscheiden kann, ob die Familie - und als ihr Mitglied die Frau - ihrer Aufgabe als Bewahrerin der "Sitte und Tradition" gerecht wird, ist mit den Rechten der Frau als Individuum unvereinbar.

Die mögliche Ausdehnung der Rechte von Frauen durch den Verweis auf internationales Recht wurde denn auch vereinzelt befürchtet, im Sinne einer Einbruchstelle für "alle feministischen Forderungen", die hätten ausgeschlossen werden können, bei Einbettung der Rechte der Frau in den "üblichen Bereich z.B. der Ehe, Arbeit oder Gesundheit". ${ }^{53}$ Andere Literaturstimmen beurteilen hingegen Art. 18 Abs. 3 AfrC und seinen Verweis grundsätzlich positiv, hätten aber der Festschreibung von konkreten Schutzmaßnahmen für Frauen in der Charta den Vorzug gegeben. ${ }^{54}$ Konkrete Schutzmaßnahmen ergeben sich aber nach der

Siehe z.B. Art. 17 IPbürgR; zu dessen Horizontalwirkung und Schutzumfang, z.B. der Unvereinbarkeit von Scheidungsverboten, Nowak (Fn. 16), Art. 17, Rn. 30 und 6. 
jetzigen Regelung in der Charta entweder aus der positiven Pflicht zum Einschreiten gegen Diskriminierungen in Art. 18 Abs. 3 AfrC oder über den "Umweg" anderer internationaler Übereinkommen, vorausgesetzt, Art. 18 Abs. 3 AfrC wird entsprechend ausgelegt. Wieder andere, grundlegende Kritik gilt ungeachtet des Verweises dem Gesamtkonzept des Art. 18 AfrC und den Vorstellungen von den Rechten der Frau, die sich aus der Analyse des Kontextes des Art. 18 Abs. 3 AfrC offenbaren, der sich im übrigen mit der Familie, Kindern, Alten und Behinderten beschäftigt. ${ }^{55}$ Die Auffassung von Frauen als traditionell schutzbedürftiger Gruppe werde hiermit zementiert. ${ }^{56}$

$\mathrm{Zu}$ unterstreichen bleibt, dass ungewiss ist, ob die Organe in der Praxis eine entsprechende Ausdehnung des Schutzes durch die Einbeziehung internationaler Vorschriften und ein konkrete Bindung der Mitgliedstaaten tatsächlich annehmen werden. Mitunter wurde die Haltung der - überwiegend männlichen - Kommissionsmitglieder gegenüber den Rechten von Frauen skeptisch beurteilt. ${ }^{57}$ Andererseits lässt die Haltung der Kommission bei den bisher vorgenommenen Prüfungen der Staatenberichte ${ }^{58}$ (Art. 62 AfrC) zur Situation der Frauen und das Insistieren auf den staatlichen Maßnahmen zur Beseitigung von Diskriminierungen gegenüber Frauen auf Aufgeschlossenheit hoffen, ebenso wie die Aussagen von Kommissionsmitgliedern, die Situation afrikanischer Frauen verbessern zu wollen. ${ }^{59}$

Zusammenfassend folgt hieraus, dass aufgrund der hier vertretenen Auslegung des "renvois" durch die Einbeziehung internationalen Rechts den Gefahren, die für die Rechte von Frauen aus dem Familienschutz und der Berufung auf die Funktion der Familie als Bewah-

und geistiges Wohlbefinden" zustanden. Ein fast wortgleicher Passus befindet sich im heutigen Art. 18 Abs. 4 AfrC (Schutz der "Alten und Behinderten"). Hiermit wäre allerdings eine Ungleichheit der Geschlechter festgeschrieben worden, die sich zudem auf körperliche und intellektuelle Gründe als Rechtfertigung gestützt hätte, hierzu Ouguergouz (Fn. 42), S. 126 (Anm. 177).

Elmadmad (Fn. 12), S. 79, zur durch die Formulierung des Art. 18 Abs. 3 und 4 AfrC ersichtlichen Verbindung mit den genannten Personen.

56 Deutlich wird die Einordnung von Frauen insoweit bei Balanda, African Charter on Human and Peoples' Rights, in: Ginther / Benedek (Hrsg.), New Perspectives and Conceptions of International Law, S. 141.

Ankumah, The African Commission on Human and Peoples' Rights, Practice and Procedures (The Hague et al. 1996), S. 157 f., unter Bezugnahme auf öffentliche, frauenfeindliche Äußerungen von Mitgliedern der AfrKMV.

Hierzu Wittinger (Fn. 7), S. 205 ff. m.w.N.

Examination of the State Reports, Report of The Gambia in Accordance with Art. 62 of the African Charter on Human and Peoples' Rights, Banjul Ministry of Justice, Doc. CAG/C/144/ Vol.3/ (43), S. 31; siehe auch die Ansicht des AfrKMV-Mitgliedes Umozurike zur Interpretation des Verweises in Art. 18 Abs. 3 AfrC i.S. einer Anwendbarkeit des internationalen Rechts, hierzu Benedek / Heinz, Les systèmes régionaux de protection des droits de l'homme en Afrique, Amérique et en Europe, Troisième Conférence afro-américano-europeénne, Actes de la Conférence, Friedrich Naumann Stiftung (Brüssel 1992), S. 19. 
rerin der Traditionen erwachsen können, wirksam begegnet werden kann. Allerdings bleibt der "Preis der Theorie" durch die Auslegungsfähigkeit des Verweises.

\section{Fazit}

Die Afrikanische Charta steht in einem Spannungsverhältnis aus afrikanischer Tradition und dem internationalen Recht. Dieses Spannungsverhältnis wird relevant, wenn Familienstrukturen von der traditionellen Großfamilie abweichen oder Familien aus einem sonstigem Grund "missliebig" sind und wenn Frauen im Konfliktfall ihre Rechte als Individuum gegenüber der Familie als "Bewahrerin der Sittlichkeit und der traditionellen Werte" einfordern und afrikanische Werte, die sich z.B. im Gewohnheitsrecht niederschlagen, damit in Frage stellen. Über die Heranziehung internationalen Rechts als Interpretationsregel in Art. $60 \mathrm{AfrC}$ und vor allem über die direkte Anwendbarkeit internationaler Vorschriften durch den Verweis in Art. 18 Abs. 3 AfrC bietet sich die Möglichkeit, Gefahren für Familien und für das Individuum durch die vorgegebene Einbindung der Charta in den internationalen Menschenrechtsschutz mit Hilfe desselben zu bannen. Es wird Aufgabe der Afrikanischen Menschenrechtskommission und des zukünftigen Afrikanischen Gerichtshofes sein, diese Möglichkeiten der Charta auszuschöpfen. 